BMJ Open

Diabetes

Research

\& Care

\title{
Oxidized LDL, insulin sensitivity and beta-cell function in newborns
}

Fang Fang, ${ }^{1,2}$ Anne Monique Nuyt, ${ }^{3}$ Carole Garofalo, ${ }^{3}$ Jun Zhang, ${ }^{1}$ Pierre Julien, ${ }^{4}$ William Fraser, ${ }^{3,5}$ Emile Levy, ${ }^{3}$ Zhong-Cheng Luo (1) ${ }^{1,2}$

To cite: Fang F, Nuyt AM, Garofalo $\mathrm{C}$, et al. Oxidized LDL, insulin sensitivity and beta-cell function in newborns. BMJ Open Diab Res Care 2021;9:e001435. doi:10.1136/ bmjdrc-2020-001435

Received 3 April 2020 Revised 1 December 2020 Accepted 9 January 2021

Check for updates

\section{(c) Author(s) (or their} employer(s)) 2021. Re-use permitted under CC BY-NC. No commercial re-use. See rights and permissions. Published by BMJ.

For numbered affiliations see end of article.

Correspondence to Dr Zhong-Cheng Luo; zcluo@lunenfeld.ca

\section{ABSTRACT}

Introduction 0xidized low-density lipoprotein (0xLDL), a biomarker of oxidative stress, itself possesses proatherogenic and proinflammatory effects. Elevated circulating OxLDL levels have been consistently associated with insulin resistance and diabetes in adults. We sought to assess whether OxLDL may be associated with insulin sensitivity and beta-cell function in early life.

Research design and methods In a birth cohort study, we assessed cord plasma $0 x L D L$ concentration and OxLDL to total LDL ratio in relation to glucose to insulin ratio (an indicator of fetal insulin sensitivity), proinsulin to insulin ratio (an indicator of fetal beta-cell function), and leptin and adiponectin concentrations in 248 singleton newborns. Results Cord plasma 0xLDL concentration was positively correlated with glucose to insulin ratio $(r=0.24, p<0.001)$ and proinsulin to insulin ratio $(r=0.20, p<0.001)$ and was not correlated with leptin or adiponectin. Adjusting for maternal and neonatal characteristics, each log unit increase in cord plasma 0xLDL concentration was associated with a $25.8 \%(95 \% \mathrm{Cl} 12.8 \%$ to $40.3 \%)$ increase in glucose to insulin ratio and a 19.0\% (95\% Cl $6.8 \%$ to $32.9 \%$ ) increase in proinsulin to insulin ratio, respectively. Similar associations were observed for cord plasma $0 x L D L$ to $L D L$ ratio in relation to cord plasma glucose to insulin ratio and proinsulin to insulin ratio. Conclusions Higher 0xLDL levels were associated with lower fetal beta-cell function (higher proinsulin to insulin ratio) but higher insulin sensitivity (higher glucose to insulin ratio). The study is the first to demonstrate that OxLDL may affect glucose metabolic health in early life in humans.

\section{INTRODUCTION}

The rising epidemic of metabolic syndrome and related disorders such as type 2 diabetes has become a major public health burden. ${ }^{1-3}$ Increasing evidence points to the role of exposures in early life in developmentally 'programming' the vulnerability to cardiometabolic disease risks. ${ }^{45}$ The mechanisms remain largely unknown. Evidence from basic science research suggests that oxidative stress-the loss of balance between the pro-oxidative and antioxidative forces-may play an important role in metabolic programming. ${ }^{6} 7$ Oxidative stress tips the balance of pro-oxidative versus antioxidative forces toward the abundance of numerous oxidation molecules,

\section{Significance of this study}

What is already known about this subject?

- 0xidized low-density lipoprotein (0xLDL), a biomarker of oxidative stress, itself possesses proatherogenic and proinflammatory effects.

- Elevated circulating OxLDL levels have been consistently associated with insulin resistance and diabetes in adults, but it is unknown whether OxLDL may affect metabolic health in early life.

What are the new findings?

- Higher cord blood OxLDL concentrations were associated with lower beta-cell function (higher proinsulin to insulin ratio) but higher insulin sensitivity (higher glucose to insulin ratio) in newborns.

- Cord blood OxLDL was not correlated with leptin or adiponectin.

- The study is the first to demonstrate that OxLDL may affect glucose metabolic health in early life in humans.

How might these results change the focus of research or clinical practice?

- OxLDL may affect fetal beta-cell function and more studies are warranted to validate this novel finding.

- If further studies validate the impact of OxLDL on glucose metabolic health in fetal life, it may become a molecular target for interventions to safeguard healthy early life development.

and some of them may have developmental toxicity. Oxidized low-density lipoprotein (OxLDL), a product of lipoprotein oxidation and biomarker of oxidative stress, ${ }^{8}$ is of particular interest since it can induce endothelial cell activation and macrophage foam cell formation and exert proatherogenic and proinflammatory effects. ${ }^{9}$ Elevated circulating OxLDL levels have been associated with poor pregnancy outcomes including pre-eclampsia ${ }^{10}$ and fetal growth restriction. ${ }^{11}$ Elevated circulating OxLDL concentrations are strongly predictive of an increased risk of metabolic syndrome and hyperglycemia in adults, ${ }^{12}$ suggesting that OxLDL may affect insulin sensitivity or beta-cell function. The developing fetus is likely more vulnerable 
to the deleterious effects of OxLDL. It has not been explored whether OxLDL may affect insulin sensitivity and beta-cell function in early life. The present study sought to evaluate whether circulating OxLDL levels are associated with insulin sensitivity and beta-cell function in newborns.

\section{RESEARCH DESIGN AND METHODS}

\section{Subjects and specimens}

This study was based on a prospective singleton birth cohort. $^{13}$ The original pregnancy cohort recruited 339 women bearing a singleton non-malformation fetus without pre-existing diabetes, chronic hypertension, endocrine disorders or other severe maternal illnesses at 24-28 weeks of gestation between August 2006 and December 2008 in three obstetric care centers in Montreal. There were 31 subjects lost to follow-up and 60 subjects without maternal and cord plasma specimen available for assays of OxLDL and lipids, leaving 248 subjects $(73 \%)$ in the final study cohort. Written informed consent was obtained from all participants for the use of data and specimens in research on pregnancy and infant health.

Maternal venous blood specimens were collected at 24-28 weeks of gestation. Cord venous blood specimens were collected immediately after safe delivery of the infant and before the delivery of the placenta. A tube of EDTA blood sample was specifically collected for assays of OxLDL by adding $0.1 \%$ butylated hydroxytoluene to prevent oxidation after specimen collection. A fluoridecoated (for protecting glucose) tube of blood sample was specifically collected for glucose assays. All specimens were kept on ice and centrifuged within $30 \mathrm{~min}$ after collection. Plasma samples were stored in multiple aliquots at $-80^{\circ} \mathrm{C}$ until biomarker assays. Assays of all biomarkers were completed in 12-24 months after the specimen collection. There were no significant correlations between specimen storage (at $-80^{\circ} \mathrm{C}$ ) duration and biomarker measurement values (correlation coefficients: $0.0-0.2$, all $\mathrm{p}>0.1$ ).

\section{Biochemical assays}

Plasma lipids (triglycerides, high-density lipoprotein (HDL) cholesterol and LDL cholesterol, $\mathrm{mmol} / \mathrm{L}$ ) were measured by an automated multianalyzer (Unicel DXC 880i; Beckman Coulter, Brea, California, USA). The intra-assay and interassay coefficients of variation (CVs) were lower than $3.0 \%$.

Plasma OxLDL (U/L) was measured by mAb-4E6based competition ELISA (Cat No 10-1143-01; Mercodia, Uppsala, Sweden). The mAb-4E6 antibody is directed against a conformational epitope in the apolipoprotein B-100 moiety of LDL that is generated as a consequence of the substitution of 60 lysine residues of apolipoprotein B-100 with aldehydes. The intra-assay and interassay CVs were $6.3 \%$ and $9.1 \%$, respectively.
As reported previously, plasma glucose $(\mathrm{mmol} / \mathrm{L})$ was measured by an automated glucose oxidase method, insulin $(\mathrm{pmol} / \mathrm{L})(1 \mu \mathrm{U} / \mathrm{mL}=6 \mathrm{pmol} / \mathrm{L})$ by an ultrasensitive chemiluminescent immunometric assay, and proinsulin (pmol/L) by a quantitative ELISA kit. ${ }^{13}$ The intra-assay and interassay CVs were in the $2 \%-6 \%$ range. Plasma leptin $(\mathrm{ng} / \mathrm{mL})$ was measured by a human leptin immunoassay kit and total adiponectin $(\mu \mathrm{g} / \mathrm{mL})$ by a human adiponectin immunoassay kit. ${ }^{14}$ The intra-assay and interassay CVs were in the $5.8 \%-10.4 \%$ range.

\section{Outcomes}

The primary outcomes were cord plasma glucose to insulin ratio (higher values indicate better fetal insulin sensitivity) and proinsulin to insulin ratio (higher values indicate worse fetal beta-cell function). ${ }^{13} 15$ We also assessed cord plasma concentrations of leptin and adiponectin, which are important adipokines in the regulation of insulin sensitivity. ${ }^{16}$

\section{Statistical analysis}

Median and mean \pm SE were presented for biomarker variables. Biomarker data were log-transformed in all comparisons. Partial correlation coefficients were calculated in assessing the associations of maternal and cord plasma OxLDL concentrations and OxLDL to LDL ratio with glucose metabolic health biomarkers in newborns, adjusting for gestational age at blood sampling and delivery. Generalized linear models were applied to assess the associations, adjusting for maternal and neonatal characteristics, including maternal ethnicity (white, others), age ( $<35$ years, $\geq 35$ years), prepregnancy body mass index (SD score), plasma glucose concentration in 1-hour $50 \mathrm{~g}$ oral glucose tolerance test at 24-28 weeks of gestation (SD score), parity (primiparous, yes/no), maternal smoking (yes/no) and alcohol use (yes/no), gestational hypertension (yes/no), bacterial vaginosis or treatment for infection during pregnancy (yes/no), mode of delivery (cesarean section: yes/no), duration of labor, fasting status at delivery, infant sex, gestational age and birth weight (z score, according to the Canadian sex-specific and gestational age-specific birthweight standards ${ }^{17}$ ). Data analyses were conducted using Statistical Analysis System (SAS) V.9.2. Two-tailed $p$ values $<0.025$ were considered statistically significant, accounting for two primary associations of interest: cord plasma OxLDL in relation to glucose to insulin ratio and proinsulin to insulin ratio (Bonferroni-adjusted $\mathrm{p}$ cut-off $=0.05 / 2=0.025$ ).

There were 26 newborns of mothers with gestational diabetes according to the American Diabetes Association's 2003 diagnostic criteria ${ }^{18}$ and 3 newborns of mothers with pre-eclampsia (de novo gestational hypertension with proteinuria in pregnancy). Sensitivity analyses were conducted to examine whether the findings were similar if the analyses were restricted to term infants born to mothers without pre-eclampsia and gestational 
Table 1 Maternal (24-28 weeks of gestation) and cord plasma concentrations of triglycerides, HDL, LDL, OxLDL, and OxLDL to $L D L$ ratio and surrogate indices of insulin sensitivity and beta-cell function in newborns in a singleton birth cohort $(N=248)$

\begin{tabular}{|c|c|c|c|c|c|}
\hline & $\begin{array}{l}\text { Maternal } \\
\text { plasma }\end{array}$ & & $\begin{array}{l}\text { Cord } \\
\text { plasma }\end{array}$ & & $\begin{array}{l}\text { P } \\
\text { value* }\end{array}$ \\
\hline Biomarker & Median & Mean \pm SE & Median & Mean \pm SE & \\
\hline Triglycerides, mmol/L & 2.15 & $2.22 \pm 0.05$ & 0.35 & $0.43 \pm 0.02$ & $<0.001$ \\
\hline $\mathrm{HDL}, \mathrm{mmol} / \mathrm{L}$ & 1.94 & $1.98 \pm 0.03$ & 0.64 & $0.70 \pm 0.02$ & $<0.001$ \\
\hline LDL, mmol/L & 3.08 & $3.21 \pm 0.06$ & 0.73 & $0.80 \pm 0.03$ & $<0.001$ \\
\hline OxLDL, U/L & 1434.8 & $1815.5 \pm 112.7$ & 556.4 & $905.5 \pm 84.7$ & $<0.001$ \\
\hline OxLDL:LDL, U/mmol & 468.9 & $591.4 \pm 29.2$ & 790.7 & $1278.0 \pm 141.2$ & $<0.001$ \\
\hline Glucose, mmol/L & & & 4.57 & $4.64 \pm 0.06$ & \\
\hline Insulin, pmol/L & & & 27.2 & $36.7 \pm 2.4$ & \\
\hline Glucose:insulin ratio (mmol:pmol) & & & 0.16 & $0.23 \pm 0.01$ & \\
\hline Proinsulin:insulin ratio (pmol:pmol) & & & 0.56 & $0.74 \pm 0.06$ & \\
\hline
\end{tabular}

Glucose $1 \mathrm{mmol} / \mathrm{L}=18 \mathrm{mg} / \mathrm{dL}$; insulin $1 \mathrm{mU} / \mathrm{L}=6 \mathrm{pmol} / \mathrm{L}$.

${ }^{*} \mathrm{P}$ values in t-tests for differences in cord versus maternal plasma concentrations in log-transformed data.

HDL, high-density lipoprotein; LDL, low-density lipoprotein; OxLDL, oxidized low-density lipoprotein.

diabetes, or if the analyses were restricted to the newborns of fasting mothers at delivery $(n=89)$.

\section{RESULTS}

Most maternal and neonatal characteristics (ethnicity, age, prepregnancy body mass index, plasma glucose concentration in 1-hour $50 \mathrm{~g}$ oral glucose tolerance test at 24-28 weeks of gestation, parity, smoking and alcohol use, infant sex, gestational age, birth weight and mode of delivery) in this birth cohort have been described previously. ${ }^{13}$ Here, we only described the characteristics not reported previously but relevant to the present study. There were $38(15.3 \%)$ women with bacterial vaginosis or any treatment for infection during pregnancy. There were $41(16.5 \%)$ infants delivered by planned cesarean section without labor and $29(11.7 \%)$ by cesarean section after labor. The mean duration of labor was $8.2 \pm 0.4$ hours. There were 89 women fasted during delivery.

Plasma concentrations of all lipids (triglycerides, HDL cholesterol and LDL cholesterol) and OxLDL were substantially lower in cord versus maternal plasma (table 1). However, OxLDL to LDL ratios were more than two times higher in cord versus maternal plasma (1278.0 $\pm 141.2 \mathrm{U} / \mathrm{mmol}$ vs $591.4 \pm 29.2 \mathrm{U} / \mathrm{mmol}, \mathrm{p}<0.001)$. Descriptive statistics (median, mean $\pm \mathrm{SE}$ ) on cord plasma glucose and insulin concentration, glucose to insulin ratio, and proinsulin to insulin ratio are presented in table 1. Descriptive statistics on cord plasma leptin and adiponectin concentrations have been described previously. ${ }^{14}$

Comparing cord blood biomarkers in fasting $(n=89)$ versus non-fasting $(\mathrm{n}=159)$ subjects at delivery, there were significant differences in glucose, insulin, proinsulin and adiponectin concentrations and glucose to insulin ratios, while the concentrations of triglycerides, LDL, OxLDL and leptin were similar (table 2). Comparing infants born with labor $(\mathrm{n}=207)$ with those born without labor $(n=41)$, cord plasma concentrations were higher for triglycerides (mean \pm SE: $0.45 \pm 0.02 \mathrm{mmol} / \mathrm{L}$ vs $0.31 \pm 0.03 \mathrm{mmol} / \mathrm{L}, \mathrm{p}=0.003$ ), lower for HDL cholesterol $(0.68 \pm 0.02 \mathrm{mmol} / \mathrm{L}$ vs $0.82 \pm 0.07 \mathrm{mmol} / \mathrm{L}, \mathrm{p}=0.003)$, and marginally lower for LDL cholesterol $(0.77 \pm 0.03 \mathrm{mmol} / \mathrm{L}$ vs $0.91 \pm 0.11 \mathrm{mmol} / \mathrm{L}, \mathrm{p}=0.07$ ), but higher for $\mathrm{OxLDL}$ concentration $(954.3 \pm 91.5 \mathrm{U} / \mathrm{L}$ vs $570.4 \pm 62.9 \mathrm{U} / \mathrm{L}$, $\mathrm{p}=0.08)$ and $\mathrm{OxLDL}$ to LDL ratio $(1267.4 \pm 105.2 \mathrm{U} /$ $\mathrm{mmol}$ vs $727.5 \pm 79.5 \mathrm{U} / \mathrm{mmol}, \mathrm{p}=0.07)$.

Scatter plots (data in log scale) demonstrated clear positive correlations of cord plasma OxLDL concentration and OxLDL to LDL ratio with glucose to insulin ratio (regression test for trends, $\mathrm{p}<0.001$ ) and proinsulin to insulin ratio $(\mathrm{p}=0.002)$ in newborns (figure 1$)$.

Maternal and cord plasma OxLDL concentrations were strongly positively correlated $(\mathrm{r}=0.57, \mathrm{p}<0.001)$ (table 3 ). Cord plasma OxLDL concentration and OxLDL to LDL ratio were positively correlated with glucose to insulin ratio $(r=0.24$ and $r=0.23$, all $p<0.001)$ and proinsulin to insulin ratio $(r=0.20$ for both, $\mathrm{p}=0.002)$, but not correlated with leptin and adiponectin (all $\mathrm{p}>0.2$ ). Maternal plasma OxLDL concentration and OxLDL to total LDL ratio were also positively correlated with cord plasma proinsulin to insulin ratio $(\mathrm{r}=0.17$ for both, $\mathrm{p}<0.008)$, but not correlated with glucose to insulin ratio.

Adjusting for maternal and neonatal characteristics, each $\log$ unit increase in cord plasma OxLDL concentration was associated with a $25.8 \%(95 \%$ CI $12.8 \%$ to $40.3 \%)$ increase in cord plasma glucose to insulin ratio $(\mathrm{p}<0.001)$ and a $19.0 \%$ (95\% CI $6.8 \%$ to $32.9 \%$ ) increase in proinsulin to insulin ratio $(\mathrm{p}=0.002)$, respectively (table 4$)$. Similar effects were observed for cord plasma OxLDL to LDL ratio: each $\log$ unit increase in OxLDL to LDL ratio was associated with a $22.4 \%(10.1 \%-36.2 \%)$ increase in glucose to insulin ratio $(\mathrm{p}<0.001)$ and a $17.0 \%(5.3 \%-30.0 \%)$ increase 
Table 2 Cord plasma biomarkers in the newborns of fasting and non-fasting mothers $(\mathrm{N}=248)$

\begin{tabular}{|c|c|c|c|c|c|}
\hline \multirow[b]{2}{*}{ Biomarker* } & \multirow{2}{*}{$\begin{array}{l}\begin{array}{l}\text { Fasting } \\
(\mathrm{n}=89)\end{array} \\
\text { Median } \\
\end{array}$} & \multirow[b]{2}{*}{ Mean $\pm S E$} & \multicolumn{2}{|c|}{$\begin{array}{l}\text { Non-fasting } \\
(\mathrm{n}=159)\end{array}$} & \multirow[b]{2}{*}{ P value* } \\
\hline & & & Median & Mean \pm SE & \\
\hline Triglycerides, mmol/L & 0.35 & $0.43 \pm 0.03$ & 0.35 & $0.43 \pm 0.02$ & 0.47 \\
\hline $\mathrm{HDL}, \mathrm{mmol} / \mathrm{L}$ & 0.68 & $0.75 \pm 0.04$ & 0.63 & $0.68 \pm 0.02$ & 0.09 \\
\hline LDL, mmol/L & 0.73 & $0.87 \pm 0.07$ & 0.73 & $0.76 \pm 0.02$ & 0.69 \\
\hline OxLDL, U/L & 533.0 & $791.9 \pm 127.4$ & 562.2 & $943.5 \pm 97.8$ & 0.27 \\
\hline OxLDL:LDL, U/mmol & 765.2 & $1075.7 \pm 146.3$ & 796.7 & $1231.4 \pm 113.0$ & 0.30 \\
\hline Glucose, mmol/L & 3.95 & $4.03 \pm 0.08$ & 4.70 & $4.98 \pm 0.07$ & $<0.0001$ \\
\hline Insulin, pmol/L & 24.10 & $32.13 \pm 2.59$ & 32.60 & $44.78 \pm 4.77$ & 0.009 \\
\hline Glucose:insulin ratio & 0.12 & $0.18 \pm 0.02$ & 0.20 & $0.27 \pm 0.02$ & $<0.0001$ \\
\hline Proinsulin, pmol/L & 15.90 & $18.28 \pm 1.19$ & 12.70 & $17.54 \pm 1.19$ & 0.02 \\
\hline Proinsulin:insulin ratio & 0.51 & $0.66 \pm 0.05$ & 0.56 & $0.78 \pm 0.08$ & 0.10 \\
\hline Leptin & 24.80 & $42.91 \pm 5.19$ & 27.14 & $38.69 \pm 3.09$ & 0.95 \\
\hline Adiponectin & 18.14 & $19.66 \pm 0.75$ & 20.28 & $21.20 \pm 0.66$ & 0.04 \\
\hline
\end{tabular}

$P$ values in bold, $P<0.05$.

${ }^{*} P$ values in Wilcoxon tests for differences in fasting versus non-fasting subjects.

HDL, high-density lipoprotein; LDL, low-density lipoprotein; OxLDL, oxidized low-density lipoprotein.

in proinsulin to insulin ratio $(\mathrm{p}=0.004)$, respectively. Similarly, higher maternal plasma OxLDL concentrations and OxLDL to LDL ratios were associated with higher cord plasma glucose to insulin ratios and higher proinsulin to insulin ratios, although some of these associations were not
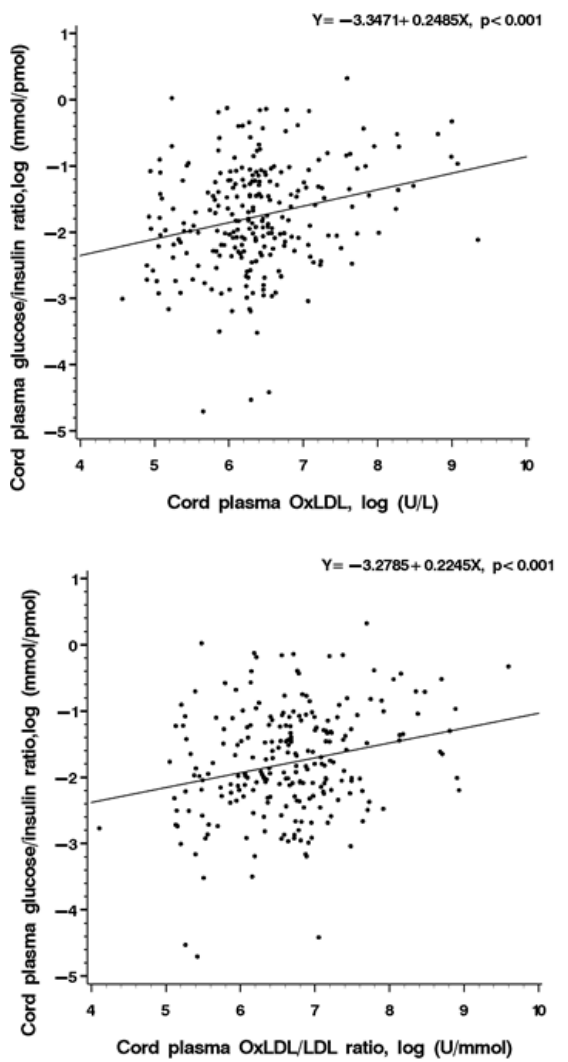

Figure 1 Scatter plots (in log scale) illustrating the positive correlations of cord plasma OxLDL concentration and OxLDL to LDL ratio with glucose to insulin ratio and proinsulin to insulin ratio in newborns. LDL, low-density lipoprotein; OxLDL, oxidized low-density lipoprotein.

statistically significant after the adjustments ( $\mathrm{p}$ values in the borderline range of $0.05-0.10)$.

Comparing gestational diabetic $(\mathrm{n}=26)$ versus euglycemic pregnancies, maternal plasma triglycerides levels were significantly higher $(\mathrm{p}<0.01)$, while there were
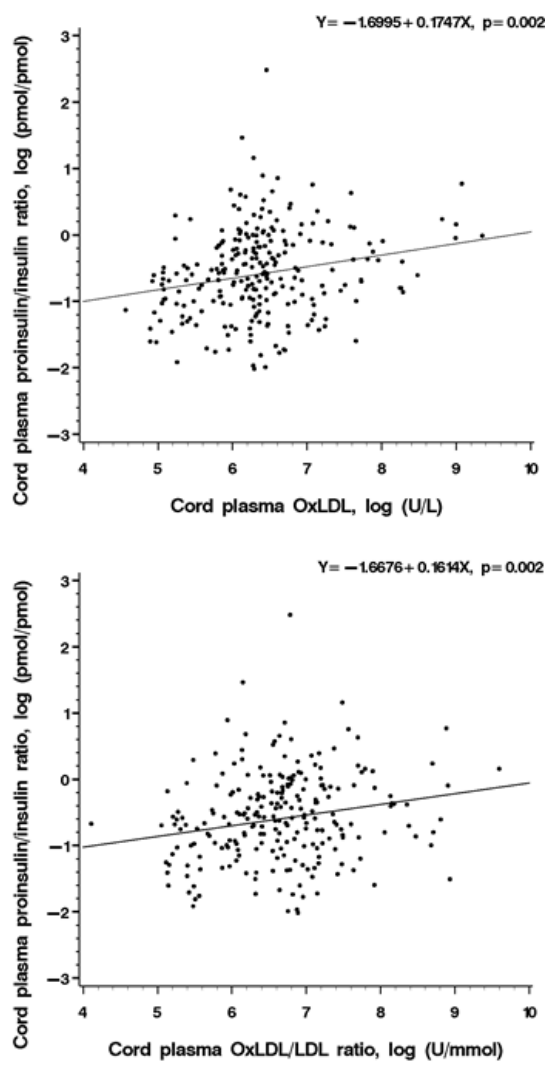
Table 3 Partial correlation coefficients of maternal and cord plasma OxLDL concentration and OxLDL to LDL ratio with metabolic health biomarkers in newborns in a singleton birth cohort $(\mathrm{N}=248)$

\begin{tabular}{lcccc}
\hline & Maternal plasma & \multicolumn{3}{c}{ Cord plasma } \\
\hline Cord plasma & OxLDL & OxLDL:LDL & OxLDL & OxLDL:LDL \\
\hline OxLDL & $\mathbf{0 . 5 7 \dagger}$ & $\mathbf{0 . 5 3 \dagger}$ & - & \\
OxLDL:LDL ratio & $\mathbf{0 . 5 7 \dagger}$ & $\mathbf{0 . 4 7 \dagger}$ & $\mathbf{0 . 8 9 \dagger}$ & \\
Glucose:insulin ratio & 0.12 & 0.04 & $\mathbf{0 . 2 4 \dagger}$ & $\mathbf{0 . 2 3} \dagger$ \\
Proinsulin:insulin ratio & $\mathbf{0 . 1 7}$ & $\mathbf{0 . 1 7}$ & $\mathbf{0 . 2 0 \ddagger}$ & $\mathbf{0 . 2 0}$ \\
Leptin & -0.06 & -0.02 & -0.11 & -0.10 \\
Adiponectin & -0.14 & -0.13 & 0.01 & 0.02 \\
\hline
\end{tabular}

Correlation coefficients in bold, $\mathrm{p}<0.05$.

${ }^{*} \mathrm{P}<0.025$.

$\dagger \mathrm{P}<0.001$

$\ddagger \mathrm{P}<0.01$.

§earson partial correlation coefficients of log-transformed biomarker data adjusting for gestational age at maternal and cord blood sampling.

LDL, low-density lipoprotein; OxLDL, oxidized low-density lipoprotein.

no significant differences in maternal or cord plasma concentrations of OxLDL, LDL cholesterol and HDL cholesterol and OxLDL to LDL ratios (all $\mathrm{p}>0.1$; data not shown). There were no significant differences in cord plasma OxLDL concentrations and OxLDL to LDL ratios comparing cesarean $(n=70)$ versus vaginal deliveries, smallfor-gestational age (SGA) $(<10$ th percentile in birth weight for sex and gestational age according to the Canadian fetal growth standards ${ }^{18}, \mathrm{n}=14$ ) versus non-SGA, or preterm ( $<37$ weeks, $\mathrm{n}=11$ ) versus term newborns (all $\mathrm{p}>0.1$ ).

If the analyses were restricted to term newborns of mothers excluding patients with gestational diabetes or pre-eclampsia $(n=213)$, or if the analyses were restricted to newborns of fasting mothers at delivery $(n=89)$, the findings were similar. For example, similar correlations were observed in the associations of cord plasma OxLDL and OxLDL to LDL ratio with glucose to insulin ratio and proinsulin to insulin ratio in newborns (tables 5 and 6).

\section{DISCUSSION}

\section{Main findings}

Our study is the first to demonstrate that higher cord blood OxLDL concentrations were associated with worse beta-cell function but better insulin sensitivity in newborns. The findings were consistent regardless of whether plasma OxLDL was analyzed in absolute concentration or as a ratio to total LDL, and persistent after accounting for potential confounding factors.

\section{Data interpretation and comparisons with previous findings}

Oxidative stress has been associated with beta-cell dysfunction and type 2 diabetes in adulthood. ${ }^{19}$

Table 4 Adjusted changes (\%) in plasma indices of insulin sensitivity and beta-cell function in newborns in relation to changes in maternal (24-28 weeks of gestation) or cord plasma OxLDL cholesterol concentration and OxLDL to total LDL ratio in singleton pregnancies

\begin{tabular}{|c|c|c|}
\hline & Adjusted change (\%)§ & \\
\hline Per log unit increase in: & Glucose:insulin ratio & Proinsulin:insulin ratio \\
\hline Maternal plasma OxLDL & $19.2(2.6,38.5)^{\star}$ & $15.3(-0.4,33.5)$ \\
\hline OxLDL:LDL ratio & $15.3(-0.1,32.9)$ & $18.5(3.3,35.9)^{\star}$ \\
\hline Cord plasma OxLDL & $25.8(12.8,40.3) \ddagger$ & $19.0(6.8,32.9) \dagger$ \\
\hline OxLDL:LDL ratio & $22.4(10.1,36.2) \ddagger$ & $17.0(5.3,30.0) \dagger$ \\
\hline
\end{tabular}

Regression coefficients in bold, $\mathrm{P}<0.05$.

${ }^{*} \mathrm{P}<0.025$.

$\dagger P<0.01$.

$\ddagger \mathrm{P}<0.001$.

$\S$ Adjusted for maternal glucose tolerance (plasma glucose concentration in 1-hour $50 \mathrm{~g}$ oral glucose tolerance test at 24-28 weeks of gestation, SD score), gestational hypertension, bacterial vaginosis or any treatment for infection during pregnancy, prepregnancy body mass index (SD score), ethnicity, parity, age, smoking, alcohol use, mode of delivery and duration of labor, fasting status at delivery, infant sex, gestational age and birth weight (z score). The adjusted \% change was calculated from the regression coefficient of the dependent variable $(y)$ in log scale per log unit increase in the independent variable $(x)$, because the regression coefficient $(\beta)$ represents the proportion of change in $y$ in the original scale: $\log y_{1}-\log y_{0}=\beta$, then $\log \left(y_{1} / y_{0}\right)=\beta$, thus $y_{1} / y_{0}=e^{\beta}$, and the percentage change is $\left(e^{\beta}-1\right) \times 100 \%$.

LDL, low-density lipoprotein; OxLDL, oxidized low-density lipoprotein. 
Table 5 Partial correlation coefficients§ of maternal and cord plasma OxLDL concentration and OxLDL to total LDL ratio with cord plasma metabolic health biomarkers in singleton term newborns of mothers without gestational diabetes and preeclampsia $(n=213)$

\begin{tabular}{lcccc}
\hline & Maternal plasma & \multicolumn{3}{c}{ Cord plasma } \\
\hline Cord plasma & OxLDL & OxLDL:LDL & OxLDL & OxLDL:LDL \\
\hline Glucose:insulin ratio & 0.08 & 0.06 & $\mathbf{0 . 2 7} \neq$ & $\mathbf{0 . 2 7}$ \\
Proinsulin:insulin ratio & 0.11 & 0.12 & $\mathbf{0 . 2 0}$ & $\mathbf{0 . 2 2 \dagger}$ \\
Leptin & -0.04 & -0.03 & -0.10 & -0.11 \\
Adiponectin & -0.15 & -0.14 & -0.02 & 0.02 \\
\hline
\end{tabular}

Correlation coefficients in bold, $\mathrm{P}<0.05$.

${ }^{*} \mathrm{P}<0.05$.

$\dagger P<0.01$.

$\ddagger \mathrm{P}<0.001$.

§Pearson partial correlation coefficients adjusting for gestational age at maternal and cord blood sampling; data were log-transformed for all biomarkers with skewed crude data distribution in the analyses.

LDL, low-density lipoprotein; OxLDL, oxidized low-density lipoprotein.

OxLDL - a robust biomarker of oxidative stress $^{20}$ itself possesses a number of adverse biological effects including endothelial cell activation and induction of proinflammatory cytokine production. ${ }^{9}$ Higher circulating OxLDL concentrations have been associated with increased incidence of metabolic syndrome in a prospective cohort. ${ }^{12}$ The association with insulin resistance and metabolic syndrome appears to be independent of adiposity, ${ }^{21}{ }^{22}$ suggesting an independent effect of OxLDL. Studies in animal models support the role of oxidative stress in insulin resistance. ${ }^{23}$ We observed that higher cord blood OxLDL concentrations and OxLDL to LDL ratios were correlated with higher glucose to insulin ratios (better insulin sensitivity) and higher proinsulin to insulin ratios (worse beta-cell function) in newborns, suggesting that OxLDL may affect glucose metabolic health in early life in humans. The association with insulin sensitivity was opposite the observed association in adults. This may not be a surprise, however; such opposite associations in newborns versus adults have been well documented in some metabolic health biomarkers. For example, in contrast to the negative correlation between circulating adiponectin concentration and adiposity in adults, cord blood adiponectin concentration was positively correlated with adiposity in newborns. ${ }^{24}$ We speculated that the observed positive association between OxLDL and fetal insulin sensitivity might be due to the developmental survival mechanism to compensate for lower beta-cell function to maintain glucose homeostasis.

Compared with adults, fetuses/newborns may be more prone to oxidative stress due to the relatively immature and vulnerable antioxidation defense system, such as lower activities of antioxidant enzymes. ${ }^{25}$ Consistent with this theory, we observed a twofold higher oxidation ratio of LDL in the newborns than in the mothers, although caution is warranted in data interpretation since maternal plasma LDL and OxLDL were measured at 24-28 weeks of gestation. It is unclear whether circulating OxLDL to LDL ratio might change substantially between 24-28 weeks of gestation and delivery. It is generally assumed that de novo synthesis accounts for most of the fetal cholesterol, although there may be some maternal-fetal transfers of LDL and HDL cholesterol. ${ }^{26}$ The extent

Table 6 Partial correlation coefficients§ of maternal and cord plasma OxLDL concentration and OxLDL to total LDL ratio with cord plasma metabolic health biomarkers in newborns of fasting mothers at delivery $(n=89)$

\begin{tabular}{lllcc}
\hline & Maternal plasma & \multicolumn{3}{c}{ Cord plasma } \\
\hline Cord plasma & OxLDL & OxLDL:LDL & OxLDL & OxLDL:LDL \\
\hline Glucose:insulin ratio & -0.19 & 0.06 & $\mathbf{0 . 2 1}^{\star}$ & $\mathbf{0 . 2 5} \dagger$ \\
Proinsulin:insulin ratio & $\mathbf{0 . 1 9 ^ { \star }}$ & $\mathbf{0 . 1 9 ^ { \star }}$ & $\mathbf{0 . 1 9 ^ { \star }}$ & $\mathbf{0 . 1 8}^{\star}$ \\
Leptin & 0.03 & 0.07 & -0.02 & -0.04 \\
Adiponectin & 0.02 & 0.03 & 0.09 & 0.08 \\
\hline
\end{tabular}

Correlation coefficients in bold, $\mathrm{P}<0.05$.

${ }^{*} \mathrm{P}<0.05$.

$\dagger P<0.01$.

$\ddagger \mathrm{P}<0.001$.

§Pearson partial correlation coefficients adjusting for gestational age at maternal and cord blood sampling; data were log-transformed for all biomarkers with skewed crude data distribution in the analyses.

LDL, low-density lipoprotein; OxLDL, oxidized low-density lipoprotein. 
of maternal contribution to fetal circulating LDL and OxLDL levels is unclear.

We found a negative association between OxLDL and fetal/neonatal beta-cell function. This is consistent with emerging evidence from basic science research. Okajima et $a l^{27}$ observed that OxLDL might impair beta-cell function by decreasing insulin secretion and pre-proinsulin mRNA expression in the pancreatic islet cells. Plaisance et $a l^{28}$ investigated the contribution of microRNAs to the adverse effects of OxLDL and observed that the expression of miR-9 was decreased whereas that of miR-21 was increased in insulin-secreting cells cultured with OxLDL particles. Upregulation of miR-21 may hamper glucoseinduced insulin secretion by modifying the expression of the components of the secretory machinery, and appropriate levels of miR-9 are required to achieve optimal insulin expression. ${ }^{29}$ OxLDL may be dangerous to beta-cells because pancreatic islets have low levels of antioxidant enzyme expression and beta-cells have high oxidative energy requirements. ${ }^{19}$

An inverse association has been observed between circulating OxLDL and adiponectin levels in adults. ${ }^{30}$ In contrast, we did not observe any significant association of cord plasma OxLDL concentration or OxLDL to total LDL ratio with cord plasma leptin and adiponectin concentrations in newborns. The associations in certain metabolic health biomarkers may be different in newborns than in adults. For example, in contrast to the positive correlation between circulating adiponectin levels and insulin sensitivity in adults, cord blood adiponectin was not associated with insulin sensitivity in newborns. ${ }^{14} 24$

We could not detect any significant difference in cord plasma OxLDL concentration or OxLDL to LDL ratio between gestational diabetic versus euglycemic pregnancies, SGA versus non-SGA, and preterm versus term births. Caution is warranted in data interpretation since the numbers of gestational diabetic pregnancies $(n=26)$, SGA $(n=14)$ and preterm births $(n=11)$ are small in our study cohort.

\section{Strengths and limitations}

The main strengths are the timely collection and processing of cord blood specimens and high-quality biomarker assays (low intra-assay and interassay $\mathrm{CVs}$ ). The main limitation is that the majority of mothers/fetuses/ newborns were not fasting at delivery and thus not in a uniform metabolic state. However, this might tend to have increased the noise variations in insulin sensitivity and beta-cell function measurements and would tend to bias the associations toward the null. Moreover, similar associations were observed in the sensitivity analysis restricted to fasting subjects. The study is observational in nature and causality could not be claimed. Lastly, the study was based on a Canadian birth cohort. Studies in other countries are required to validate the findings in other populations.

\section{CONCLUSIONS}

Higher cord blood OxLDL levels were associated with worse fetal beta-cell function, suggesting that OxLDL may affect glucose metabolic health in early life in humans. Whether this may be related to future risk of diabetes remains to be understood.

\section{Author affiliations}

${ }^{1}$ Ministry of Education-Shanghai Key Laboratory of Children's Environmental Health, Department of Pediatrics, Xinhua Hospital, Shanghai Jiao-Tong University School of Medicine, Shanghai, China

${ }^{2}$ Department of Obstetrics and Gynecology, Lunenfeld-Tanenbaum Research Institute, Prosserman Center for Population Health Research, Mount Sinai Hospital, University of Toronto, Toronto, Ontario, Canada

${ }^{3}$ Sainte-Justine University Hospital and Research Center, University of Montreal, Montreal, Québec, Canada

${ }^{4}$ Department of Medicine, Molecular and Oncologic Endocrinology and Human Genomics Research Center, CHU-Quebec Laval University Research Center, Laval University, Quebec City, Quebec, Canada

${ }^{5}$ Department of Obstetrics and Gynecology, University of Sherbrooke, Sherbrooke, Quebec, Canada

Acknowledgements We would like to acknowledge the excellent professional work of the research staff at Sainte-Justine Hospital Research Center in patient recruitment, follow-up, data management and biochemical assays.

Contributors Z-CL, AMN, WF, PJ and EL developed the research protocol. JZ contributed to study design. Z-CL, WF, AMN, CG and EL contributed to acquisition of research data. FF and Z-CL conducted the data analyses. FF drafted the manuscript. Z-CL finalized the manuscript. All authors reviewed the manuscript and approved the final version for publication. Z-CL is the guarantor of this work, has full access to all the data in the study and takes responsibility for the integrity of the data and the accuracy of the data analysis.

Funding This work was supported by research grants from the Ministry of Science and Technology of China National Key Research Program (2019YFA0802501), the Canadian Institutes of Health Research (CIHR grant \#158616 and \#79896) and the National Natural Science Foundation of China (81903323). The funders had not been involved in study design, data collection and analysis, manuscript preparation and publication decisions.

Competing interests None declared.

Patient consent for publication Not required.

Ethics approval The project was approved by the research ethics committee of the coordination center (Sainte-Justine Hospital Research Center, University of Montreal, reference number 2318) and all participating hospitals.

Provenance and peer review Not commissioned; externally peer reviewed.

Data availability statement Data are available upon reasonable request. Access to deidentified participant research data must be approved by the research ethics board on a case-by-case basis. Please contact the corresponding author (zcluo@ lunenfeld.ca) for assistance in data access request.

Open access This is an open access article distributed in accordance with the Creative Commons Attribution Non Commercial (CC BY-NC 4.0) license, which permits others to distribute, remix, adapt, build upon this work non-commercially, and license their derivative works on different terms, provided the original work is properly cited, appropriate credit is given, any changes made indicated, and the use is non-commercial. See: http://creativecommons.org/licenses/by-nc/4.0/.

ORCID iD

Zhong-Cheng Luo http://orcid.org/0000-0002-1794-1312

\section{REFERENCES}

1 Aguilar M, Bhuket T, Torres S, et al. Prevalence of the metabolic syndrome in the United States, 2003-2012. JAMA 2015;313:1973-4.

2 Mozumdar A, Liguori G. Persistent increase of prevalence of metabolic syndrome among U.S. adults: NHANES III to NHANES 1999-2006. Diabetes Care 2011;34:216-9.

3 Lim S, Shin H, Song JH, et al. Increasing prevalence of metabolic syndrome in Korea: the Korean National health and nutrition examination survey for 1998-2007. Diabetes Care 2011;34:1323-8. 
4 Gluckman PD, Hanson MA. Living with the past: evolution, development, and patterns of disease. Science 2004;305:1733-6.

5 Hales CN, Barker DJ. The thrifty phenotype hypothesis. Br Med Bull 2001;60:5-20.

6 Thompson LP, Al-Hasan Y. Impact of oxidative stress in fetal programming. J Pregnancy 2012;582748.

7 Luo ZC, Fraser WD, Julien P, et al. Tracing the origins of "fetal origins" of adult diseases: programming by oxidative stress? Med Hypotheses 2006;66:38-44.

8 Ho E, Karimi Galougahi K, Liu C-C, et al. Biological markers of oxidative stress: applications to cardiovascular research and practice. Redox Biol 2013;1:483-91.

9 Pirillo A, Norata GD, Catapano AL. Lox-1, oxLDL, and atherosclerosis. Mediators Inflamm 2013;2013:1-12.

10 Uzun H, Benian A, Madazli R, et al. Circulating oxidized low-density lipoprotein and paraoxonase activity in preeclampsia. Gynecol Obstet Invest 2005;60:195-200.

11 Maisonneuve E, Delvin E, Edgard A, et al. Oxidative conditions prevail in severe IUGR with vascular disease and Doppler anomalies. J Matern Fetal Neonatal Med 2015;28:1471-5.

12 Holvoet P, Lee D-H, Steffes M, et al. Association between circulating oxidized low-density lipoprotein and incidence of the metabolic syndrome. JAMA 2008;299:2287-93.

13 Luo Z-C, Delvin E, Fraser WD, et al. Maternal glucose tolerance in pregnancy affects fetal insulin sensitivity. Diabetes Care 2010;33:2055-61.

14 Luo Z-C, Nuyt A-M, Delvin E, et al. Maternal and fetal leptin, adiponectin levels and associations with fetal insulin sensitivity. Obesity 2013;21:210-6.

15 Gungor N, Saad R, Janosky J, et al. Validation of surrogate estimates of insulin sensitivity and insulin secretion in children and adolescents. J Pediatr 2004;144:47-55.

16 Zhang $\mathrm{H}$, Zhang $\mathrm{C}$. Adipose "talks" to distant organs to regulate insulin sensitivity and vascular function. Obesity 2010;18:2071-6.

17 Kramer MS, Platt RW, Wen SW, et al. A new and improved population-based Canadian reference for birth weight for gestational age. Pediatrics 2001;108:e35

18 American Diabetes Association. Gestational diabetes mellitus. Diabetes Care 2003;26 Suppl 1:S103-5.
19 Shah S, lqbal M, Karam J, et al. Oxidative stress, glucose metabolism, and the prevention of type 2 diabetes: pathophysiological insights. Antioxid Redox Signal 2007;9:911-29.

20 Tsimikas S. Measures of oxidative stress. Clin Lab Med 2006;26:571-90.

21 Hurtado-Roca Y, Bueno H, Fernandez-Ortiz A, et al. Oxidized LDL is associated with metabolic syndrome traits independently of central obesity and insulin resistance. Diabetes 2017;66:474-82.

22 Park K, Gross M, Lee D-H, et al. Oxidative stress and insulin resistance: the coronary artery risk development in young adults study. Diabetes Care 2009;32:1302-7.

23 Maddux BA, See W, Lawrence JC, et al. Protection against oxidative stress-induced insulin resistance in rat L6 muscle cells by mircomolar concentrations of alpha-lipoic acid. Diabetes 2001;50:404-10.

24 Basu S, Laffineuse L, Presley L, et al. In utero gender dimorphism of adiponectin reflects insulin sensitivity and adiposity of the fetus. Obesity 2009;17:1144-9.

25 Jain SK. The neonatal erythrocyte and its oxidative susceptibility. Semin Hematol 1989;26:286-300.

26 Palinski W. Maternal-Fetal cholesterol transport in the placenta: good, bad, and target for modulation. Circ Res 2009;104:569-71.

27 Okajima F, Kurihara M, Ono C, et al. Oxidized but not acetylated low-density lipoprotein reduces preproinsulin mRNA expression and secretion of insulin from HIT-T15 cells. Biochimica et Biophysica Acta (BBA) - Molecular and Cell Biology of Lipids 2005;1687:173-80.

28 Plaisance V, Abderrahmani A, Perret-Menoud V, et al. Microrna-9 controls the expression of Granuphilin/SIp4 and the secretory response of insulin-producing cells. J Biol Chem 2006;281:26932-42.

29 Roggli E, Britan A, Gattesco S, et al. Involvement of microRNAs in the cytotoxic effects exerted by proinflammatory cytokines on pancreatic beta-cells. Diabetes 2010;59:978-86.

30 Njajou OT, Kanaya AM, Holvoet P, et al. Association between oxidized LDL, obesity and type 2 diabetes in a population-based cohort, the health, aging and body composition study. Diabetes 2009;25:733-9. 